\title{
Is Complex Regional Pain Syndrome a Cause of Post-Operative Syndrome in the Lumbar Spine? - A Case Report -
}

\author{
Soo Uk Chae*, Tae Kyun Kim*, Dae Moo Shim*, Yeung Jin Kim*, Deok Hwa Choi ${ }^{\dagger}$ \\ *Department of Orthopaedic Surgery, Wonkwang Univerity Hospital, Iksan, Korea \\ ${ }^{+}$Department of Anesthesiology and Pain Medicine, Wonkwang Univerity Hospital, Iksan, Korea
}

Complex regional pain syndrome (CRPS) along with post-operative syndrome in the lumbar spine shows confusing and duplicated symptoms, and this makes it difficult to make a clear differential diagnosis. Therefore, the patient with postoperative syndrome in the lumbar spine suffers losses of time and money, and the surgeon who diagnoses and treats postoperative syndrome in the lumbar spine also agonize from the patient's losses. It is necessary to provide these patients with a multidisciplinary approach to their disease and symptoms. We diagnosed herniation of an intervertebral disc of the lumbar spine (L4/5) and we performed discetomy twice in different hospitals. However, the symptoms did not improve, so we reoperated and performed discetomy along with monosegmental fixation using pedicular screws and interbody cages. There was improvement of pre-operation symptoms, but neurogenic symptoms occurred and then progressed after the surgery. Therefore, we report here on the case of CRPS that was diagnosed with the exclusion of the causes of post-operative syndrome in the lumbar spine, and the patient was finally effectively treated with spinal cord stimulation. Although differentiating post-operative syndrome in the lumbar spine from CRPS is difficult, we recommend suspecting CRPS as the cause of post-operative syndrome in the lumbar spine and taking CRPS as the main interest in order to diagnose and treat CRPS more effectively and accurately.

Key Words: Complex regional pain syndrome, Post-operative syndrome, Lumbar spine, Spinal cord stimulation

\section{Introduction}

Complex regional pain syndrome (CRPS), along with post-operative syndrome in the lumbar spine, chronic back and leg pain (CBLP), show confusing and duplicated symptoms and so making a clear differential diagnosis is difficult. Most of these patients wander from one medical institution to another for examinations and treatments, and all this wastes the patients' time and money, as well as tying up the time of physicians. Thus, it is necessary that the mem- bers of medical faculties approach CRPS as a multiple integrated unit if the cause of post-operative syndrome in the lumbar spine is unclear after spinal surgery. CPRS is intractable chronic pain that is difficult to diagnose and treat, although there have been many investigations on this. In the realm of orthopedics, CPRS springs from trauma, nerve injury, operation, fracture, spinal cord injury and so on. CPRS requires an early diagnosis and active treatment because if treatment is delayed, then patients will suffer from irreversible changes in bones, nerves and muscles of the affected limb. CRPS is divided into CRPS type 1 and

Received Aug 17, 2009; 1st revised Sep 16, 2009; accepted Sep 28, 2009

Corresponding author: Soo Uk Chae, MD

Department of Orthopaedic Surgery, Wonkwang University Hospital,

344-2, Shinyong-dong, Iksan 570-711, Korea

Tel: +82-63-472-5100, Fax: +82-63-472-5104, E-mail: oschae68@ @anmail.net 
CRPS type 2 and these types falls under reflex sympathetic dystrophy (RSD) and causalgia, respectively ${ }^{1}$.

We diagnosed herniation of the intervertebral disc of the lumbar spine (L4/5) in a patient and we performed discetomy twice at different hospitals. However, the symptoms did not improve, so we re-operated and performed discetomy along with monosegmental fixation using pedicular screws and interbody cages. There was some improvement of the pre-operation symptoms, but then neurogenic symptoms occurred and these progressed after the surgery. We report here on a case of CPRS that was diagnosed with the exclusion of the causes of post-operative syndrome in the lumbar spine, and the patient was administered spinal cord stimula- tion (SCS), and we review the relevant literature. Here we examine if CRPS is one of the causes of post-operative syndrome in the lumbar spine.

\section{Case Report}

A 40 years old male visited our out-patient department with the chief complaints of lower back pain and right buttock pain, along with difficulty in falling asleep because of the severe pain. For the past history, he was operated on twice in different hospitals since he was injured at work where he was carrying heavy materials and he slipped with
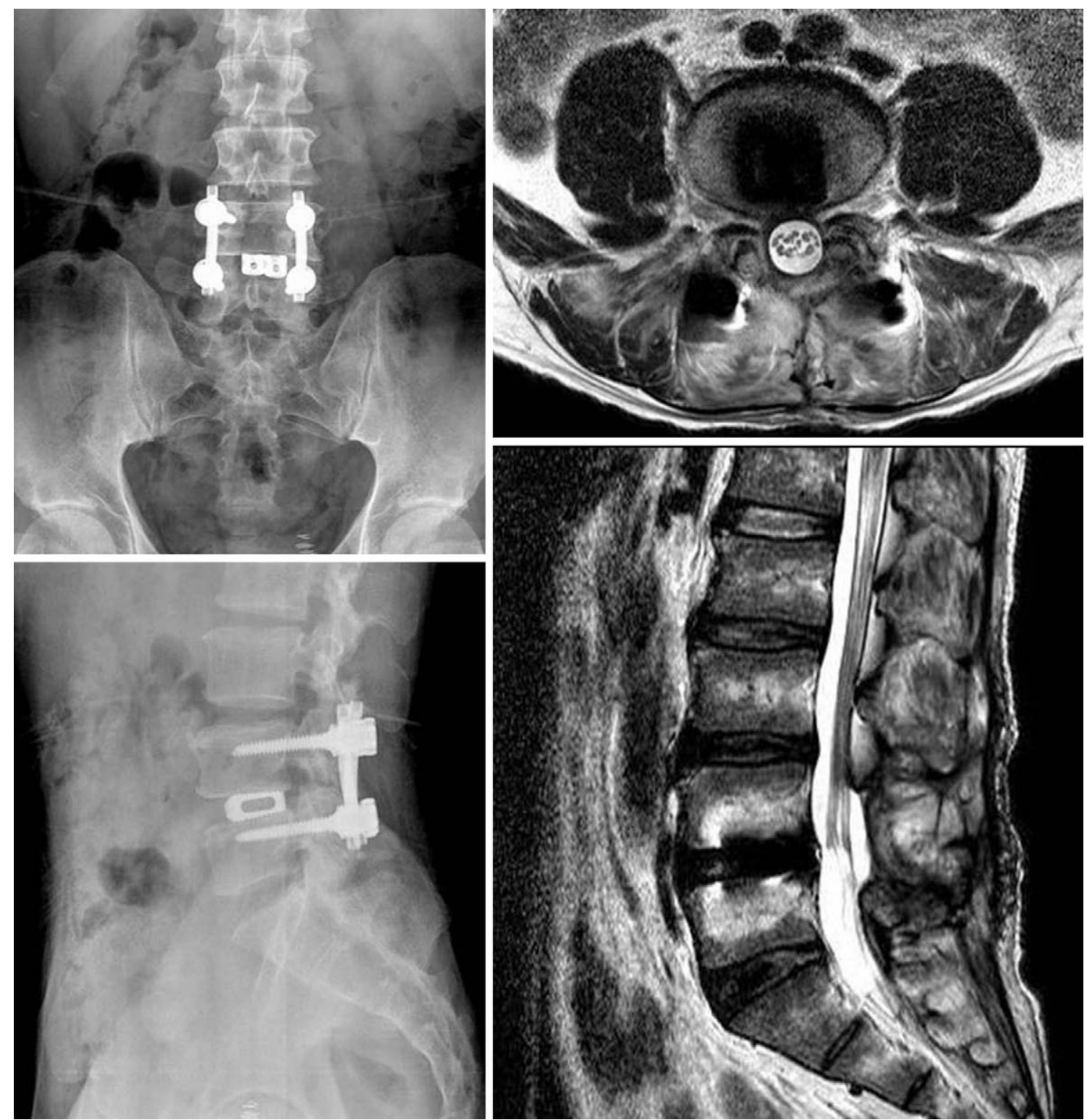

Fig. 1. The postoperative anteroposterior and lateral radiographs of the lumbar spine and the postoperative $\mathrm{T} 2$ weighted MR axial and sagittal images of the lumbar spine. 
his back bent forward. Thos occurred about 18 months before he visited the other hospital and he was operated on for the first time via endoscopic micro-discetomy. A year after that surgery, he underwent another posterior open discetomy operation in a different hospital because of his constant lower back pain. On the physical examination, it was determined that the motor and sensory nerve functions were normal and the straight leg raising test was also within the normal limits. Simple radiography of the lumbar spine was nonspecific. After being diagnosed with post-operative syndrome in the lumbar spine and being admitted into our hospital, he started exercise and physical therapy, and he received medication (anti-inflammatory drugs, analgesics and antidepressant) for three months, but he did not show any improvements. So we performed posterior interbody fusion using pedicular screws and an interbody cage (Diapason $^{\circledR}$, Stryker ${ }^{\circledR}$, Bordeaux, France) after the removal of the L4/5 disc and lamina (L4/5) (Fig. 1).

There was some improvements of the symptom until five days after the surgery, but then the patient complained of a progressive tingling sensation in both legs and difficulties in micturition and defecation with a saddle sensory decrease and a decrease of the anal sphincter tone. He showed some mild change in his symptoms several days after the surgery, but he suffered from constant neurogenic pain along with the difficulty in micturition and defecation and he was unable to walk normally because of the weakness and tingling sensation in both legs. Thus, we performed electromyography of the lower extremities and the results showed diffuse bilateral lumbosacral radiculopathies. The 3-phase bone scan showed non-specific findings. However, there was no evidence of focal disc herniation and compression of the thecal sac or nerve root, and the fixation device was in good position according to the contrast enhancement lumbar MRI findings (Fig. 1). We carried out various treatments with taking adhesive arachnoiditis, a battered nerve root or scar adhesion and the psychological compensation from the labor injury into account as the cause of the postoperative syndrome in the lumbar spine. We administered oral corticosteroid (Dexamethasone ${ }^{\circledR}$; Pacific Pharmaceuticals, Seoul, Korea) due to the neurogenic symptoms for a fixed period, and the patient continued his exercise and physical therapy, and he received medication (anti-inflammatory drugs, analgesics, and gabapentin [Neurontin ${ }^{\circledR}$; Pfizer, New York, USA]) at the same time. We also administered medication for the difficulty of micturition along with conducting uro-dynamic and bladder examinations at the urology center and we provided psychological consultation
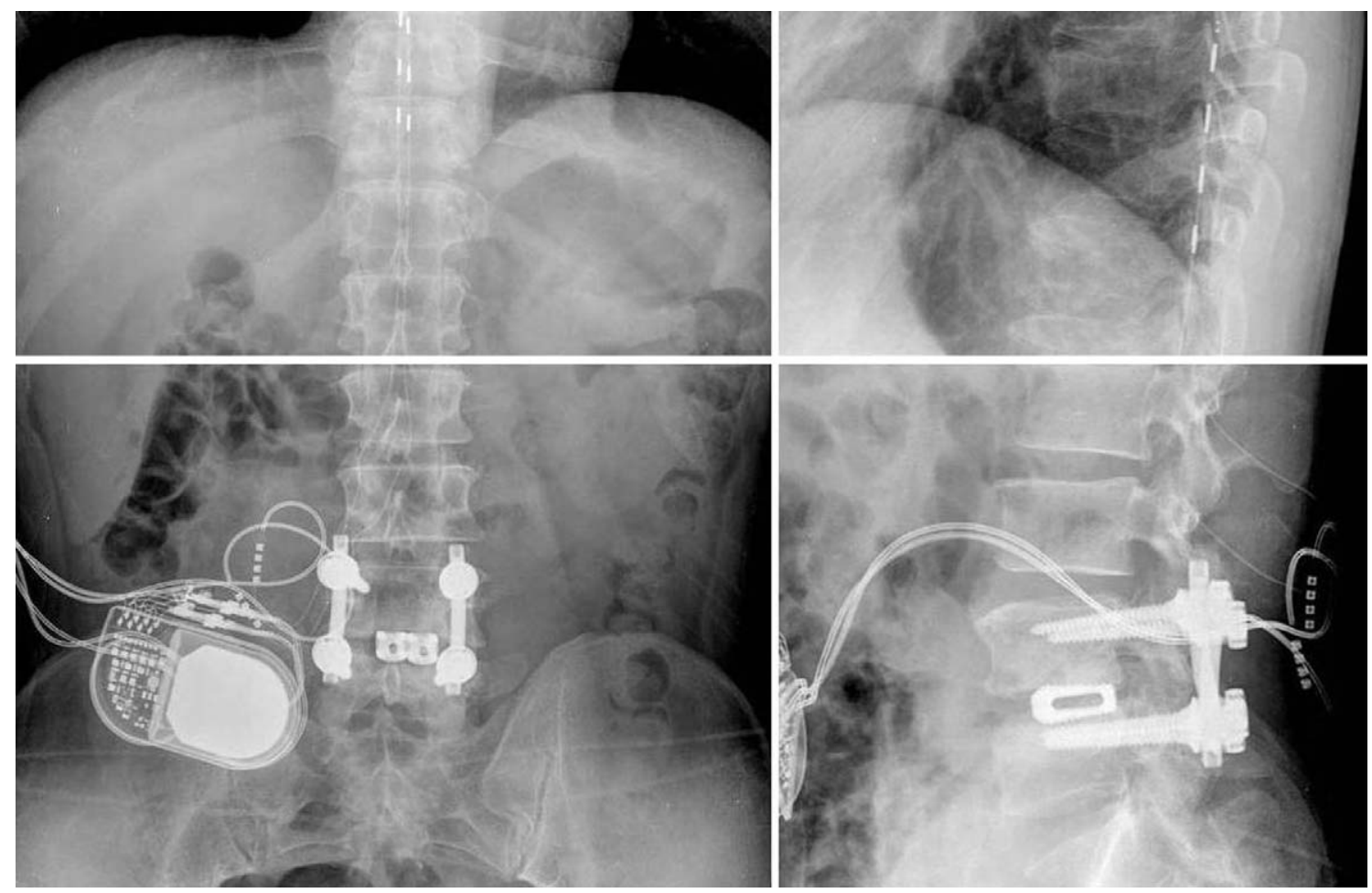

Fig. 2. The anteroposterior and lateral views of the lower thoracic spine showing the electrode tip of the spinal cord stimulator. 
and antidepressants at the neuropsychology center. Furthermore, the patient received local injection therapy that included epidural block at the pain clinic, but he did not show any satisfying improvement of the symptoms.

So we diagnosed the patients as having CRPS type 2 under the diagnostic criteria ${ }^{1,2}$ because patient showed a spontaneous tingling sensation with continuing pain in the lower back and both lower extremities (not in a specific dermatome) that was disproportionate in degree to the usual course after lumbar fusion surgery. The patient showed hyperesthesia, allodynia and dystrophic change in his feet, including saddle sensory decrease, and the motor dysfunction (weakness, tremor) of both lower extremities (below the knee joint and especially the feet). The physical examination showed edema, swelling and warmth in the feet, a decreased range of motion of the knee and ankle, and trophic change of the toenail and skin. At another pain clinic, a permanent spinal cord stimulator was implanted after a successful trial of stimulation with a temporarily (1 week) implanted electrode on the lower thoracic area (Fig. 2). According to the visual analogus scale, there was a $50 \%$ decrease in the intensity of the spontaneous tingling sensation with decreased continuing pain on a scale from 70/100 to 30 40/100, along with being able to fall asleep easily. Yet the difficulty in micturi- tion and defication, and the lower extremity tremor, weakness and hyperesthesia still existed.

\section{Discussion}

As the number of cases of spinal surgery has increased as compared to the past, the cases of post-operative syndrome in the lumbar spine, which causes pain after the spinal surgery, has also increased and there is a $15 \%$ chance of this occurring. However, it is proper to use the words "repeat or revision or refractory surgery in the lumbar operated back" since the use of the word "Failed" is inappropriate in the field of spinal surgery although the term failed back surgery syndrome (FBSS) is still frequently used in other fields of medicine $^{3}$. In our report, we used the words "post-operative syndrome in the lumbar spine" instead of "FBSS".

The cause of post-operative syndrome in the lumbar spine has been revealed from various studies. The cause of postoperative syndrome in the lumbar spine can be explained during three different periods: choosing the wrong patient and making the wrong diagnosis before the operation, the technical problems and the wrong procedure during the operation, and postoperative infection, spinal instability,

Table 1. 2004 the Internation Association for the Study of Pain proposed clinical diagnostic criteria for complex regional pain syndrome

General definition of the syndrome:

CRPS describes an array of painful conditions that are characterized by a continuing (spontaneous and/or evoked) regional pain that is seemingly disproportionate in time or degree to the usual course of any known trauma or other lesion. The pain is regional (not in a specific nerve territory or dermatome) and usually has a distal predominance of abnormal sensory, motor, sudomotor, vasomotor, and/or trophic findings. The syndrome shows variable progression over time

To make the clinical diagnosis, the following criteria must be met:

1. Continuing pain, which is disproportionate to any inciting event

2. Must report at least one symptom in three of the four following categories:

Sensory: Reports of hyperesthesia and/or allodynia

Vasomotor: Reports of temperature asymmetry and/or skin color changes and/or skin color asymmetry

Sudomotor/Edema: Reports of edema and/or sweating changes and/or sweating asymmetry

Motor/Trophic: Reports of decreased range of motion and/or motor dysfunction (weakness, tremor, dystonia) and/or trophic changes (hair, nail, skin)

3. Must display at least one sign at time of evaluation in two or more of the following categories:

Sensory: Evidence of hyperalgesia (to pinprick) and/or allodynia (to light touch and/or temperature sensation and/or deep somatic pressure and/or joint movement)

Vasomotor: Evidence of temperature asymmetry $\left(>1^{\circ} \mathrm{C}\right)$ and/or skin color changes and/or asymmetry

Sudomotor/Edema: Evidence of edema and/or sweating changes and/or sweating asymmetry

Motor/Trophic: Evidence of decreased range of motion and/or motor dysfunction (weakness, tremor, dystonia) and/or trophic changes (hair, nail, skin)

4. There is no other diagnosis that better explains the signs and symptoms

For research purposes, diagnostic decision rule should be at least one symptom in all four symptom categories and at least one sign (observed at evaluation) in two or more sign categories. 
scar adhesion, adhesive arachnoiditis and problems of the fixation device after the operation ${ }^{2}$. In this case, there were some improvements during early post-operation period, yet progressive neurogenic symptoms occurred so that we tentatively diagnosed the cause of this symptoms as adhesive arachnoiditis, a battered nerve root or scar adhesion. Despite various treatments, the symptoms did not improve, so we included CPRS according to the diagnostic criteria to diagnose and treat the patient's symptoms.

The Internation Association for the Study of Pain (IASP) named and divided CRPS into type 1 and type 2. Type 1 is similar to RSD without nerve injury after soft tissue injury, and type 2 is similar to causalgia after the nerve injury. In 2004, IASP amended the diagnosis criteria of CRPS and our case belonged to type 2 (Table 1$)^{4}$.

CRPS is a neurogenic pain that is caused by trauma, fracture, operation, nerve injury etc. It is characterized by various types of pain (tingling, allodynia, hypersentitive to pain etc), the functional loss of autonomous nerves and motor nerves, and by edema and dystrophy change. There is no single proven diagnostic tool, but taking a detailed history and understanding the chief complain along with the use of simple radiography, 3-phase bone scanning and infrared thermography can help diagnose CRPS. However, the most important thing is the clinical decision with the exclusion of other diagnosis 5 .

The principles of CRPS treatment are an early diagnosis, physical therapy and psychological consultation to stabilize the patient's mental state. There are also medicinal treatments, sympathetic nerve block, epidural block and SCS. In our case, the patient did not improve with conservative treatment such as medicinal treatment and various nerve blocks, so we treated the patients with SCS.

SCS was first introduced by Shealy et al. ${ }^{6}$ in 1967. SCS has been used for neurogenic pain such as post-operative syndrome in the lumbar spine, CRPS, arachnoiditis, cauda equina syndrome and spinal cord injury that cause CBLP. SCS eliminates autonomic dysfunction, normalizes the micro-circulation and prevents tingling and allodynia ${ }^{7}$. In the early stage, the suggested theories for the mechanism of controlling pain was the gate control theory, which explains the A- $\beta$ afferent fiber segmentation, the segmental antidromic activation, the supraspinal pain inhibition, the neuromodulator activation and the dorsal horn of the spinal cord, where gamma-amino butyric acid (GABA) was increased and adenosine was released, to ease the pain. The electrode of the spinal cord stimulator should be placed on the appropriate part of the spinal cord corresponding to the affected area of the limb with performing electrically induced paresthesia ${ }^{1.8}$. In our case, the stimulator was positioned at the lower thoracic area. A permanent spinal cord stimulator is generally implanted after a successful trial of stimulation with a temporarily implanted electrode.

The patient with post-operative syndrome in the lumbar spine suffers from lost time and money and the surgeon who diagnoses and treats post-operative syndrome in the lumbar spine also agonizes over the patient's loss. It is necessary to provide these patients with a multidisciplinary approach such referring them to a pain clinic and being in consultation from the Departments of Rehabilitation Medicine, Urology and Neuropsychology to approach the problem when the cause of post-operative syndrome in the lumbar spine is unclear. Although differentiating post-operative syndrome in the lumbar spine from CRPS is difficult, we recommend suspecting CRPS as the cause of post-operative syndrome in the lumbar spine after orthopedic surgery and taking CRPS as the main interest in order to diagnose and treat CRPS more effectively and accurately.

\section{REFERENCES}

1. Kim YC: Complex regional pain syndrome. (in The Korean Pain Society eds. Text book of pain medicine, 3rd ed. Seoul, Koonja Publishing Inc.: 75, 2007)

2. Kim BJ, Cho JT, Shin DH, Kim JH: Failed Back Surgery Syndrome: etiology and the results of the treatment. J Korean Soc Spine Surg 1999; 6: 135-140.

3. Margulies JY, Aebi M, Farcy JP: Revision spine surgery, 1st ed. St. Louis, Mosby: 1999.

4. Harden RN, Bruehl S, Stanton-Hicks M, Wilson PR: Proposed new diagnostic criteria for complex regional pain syndrome. Pain Med 2007; 8: 326-331.

5. Rho RH, Brewer RP, Lamer TJ, Wilson PR: Complex regional pain syndrome. Mayo Clin Proc 2002; 77: 174-180.

6. Shealy CN, Mortimer JT, Reswick JB: Electrical inhibition of pain by stimulation of the dorsal columns: preliminary clinical report. Anesth Analg 1967; 46: 489-491.

7. Meyerson BA, Linderoth B: Mechanisms of spinal cord stimulation in neuropathic pain. Neurol Res 2000; 22: 285292.

8. Patel S, Huang DL, Sagher O: Sympathetic mechanisms in cerebral blood flow alterations induced by spinal cord stimulation. J Neurosurg 2003; 99: 754-761. 